Ann. Génét. Sél. anim., I972, 4 (2), I59-I7r.

\title{
DESCRIPTION BIOMÉTRIQUE DE LA CROISSANCE PONDÉRALE DE POULES NAINES ET NORMALES, DISCRIMINATION PRÉCOCE SUR LE POIDS CORPOREL (DE 0 A 9 SEMAINES)

\author{
R. BARON ( $\left.{ }^{2}\right)$ \\ Station de Physiologie animale, I. N.R. A., \\ École nationale supérieure agronomique, \\ 34 - Montpellier \\ RÉSUMÉ
}

Des pesées hebdomadaires ont été effectuées de l'éclosion à 9 semaines sur un échantillon de 32 poules ( $16 d w$ et $\mathrm{I} 6 D w$ ) ; on a d'abord étudié la variabilité du poids corporel, qui est toujours plus élevée chez les $D w$ après l'âge de deux semaines. La vitesse de croissance spécifique des $D w$ est supérieure à celle des $d w$, particulièrement durant la première semaine de vie postnatale. Une fonction discriminante linéaire a été calculée, et permet de discriminer les $d w$ des $D w$ dès la $2^{e}$ semaine d'après la vitesse spécifique de croissance moyenne de la $\mathrm{I}^{\mathrm{re}}$ semaine $\left(\mathrm{X}_{1}\right)$ et le poids corporel à 2 semaines $\left(\mathrm{X}_{2}\right)$, avec un taux de mauvaise classification de 2 p. Ioo :

$$
\begin{gathered}
\mathrm{Y}=\mathrm{I0,91} \mathrm{X}_{1}+0,056 \mathrm{X}_{2} \quad\left(\mathrm{D}^{2}=45,72\right) \\
D w \text { pour } \mathrm{Y}>\mathrm{I} 5,36 \\
d w \text { pour } \mathrm{Y}<\mathrm{I4,38}
\end{gathered}
$$

\section{INTRODUC'TION}

Ce travail constitue la première étape d'une collaboration entreprise entre les Stations de Recherches avicoles de Nouzilly et du Magneraud d'une part et la Station de Physiologie animale de Montpellier d'autre part pour analyser les caractéristiques

(1) Cet article a été présenté au Symposium sur le gène de nanisme de la Poule, Tours, 4-5 mars I97I, organisé par la Station de Recherches avicoles, Nouzilly, Institut national de la Recherche agronomique.

( $\left.{ }^{2}\right)$ Un résumé anglais de cet article est déjà paru dans World's Poultry Sci. J., I971, 27, 231-232, et un résumé français dans le présent journal (Ann. Génét. Sél. anim., r97r, 3). 
de croissance des poules naines $d w$ comparées à leurs sœurs ou demi-sœurs normales Dw. On se contentera de répondre ici à la principale question posée : peut-on discriminer précocement les poules naines des normales, sachant que le choix, d'après pesées, ne peut se faire qu'après 1'âge de 9 semaines? Auparavant, on calculera et on discutera les principaux paramètres biométriques utilisables dans la description de la croissance pondérale globale de ces deux types d'animaux de l'éclosion à 9 semaines.

\section{I. — MATÉRIEL ET MÉTHODES}

\section{I. - Matériel animal}

L'étude concerne un échantillon de 32 poules ( 16 naines et $\mathrm{I} 6$ normales) issues de croisements mère normale $\times \operatorname{coq}$ hétérozygote. Ces animaux provenaient du troupeau expérimental de Jouy-en-Josas ; l'échantillon est scindé en 8 paires de sœurs et 8 paires de demi-sœurs, et on a considéré les catégories suivantes :

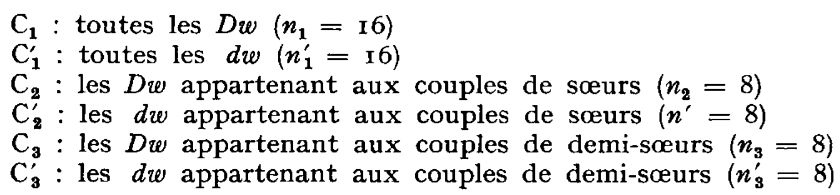

Compte tenu de la petite taille de l'échantillon, il serait illusoire de lui faire subir une nouvelle partition, par exemple de considérer la nature exacte de toutes les parentés impliquées ; c'est pourquoi les objectifs poursuivis ici auront uniquement un caractère biométrique.

Chaque poule a été pesée hebdomadairement de l'éclosion à 9 semaines (janvier-mars 1967) ; un individu sera donc caractérisé par ro variables. On dispose aussi du poids à 2 jours, mais il ne sera pas utilisé dans la discrimination $d w / D w$, sachant qu'il peut être inférieur au poids à l'éclosion (perte du sac vitellin).

\section{2. - Objectifs poursuivis et méthodes statistiques}

\section{a) Description de l'échantillon.}

Pour chacune des catégories définies plus haut, et à l'intérieur de chaque stade, on calcule les paramètres statistiques classiques : moyenne, écart-type, coefficient de variation. Ces calculs concernent trois séries de variables d'utilisation classique dans la description des phénomènes de croissance :

(i) Le poids.

On en déduira évidemment la comparaison des poids corporels intra-stades et entre catégories, et aussi l'évolution de la variabilité du poids au cours de la période o-9 semaines.

(ii) Le logarithme du poids.

La transformation logarithmique sur les poids se justifie ainsi (BARoN, I972) :

— par des raisons d'ordre statistique : stabilisation de la variance ;

- pour des raisons d'ordre biologique : la courbe Log (Poids) en fonction de l'âge est la courbe de croissance spécifique, dont on étudie la forme par :

(iii) La vitesse spécifique de croissance.

Elle est définie par :

$$
v=\frac{\mathrm{d}(\log \mathrm{X})}{\mathrm{d} t}=\frac{\mathrm{dX}}{\mathrm{X} \mathrm{d} t} .
$$


Fisher (I92I) a montré que, quelle que soit la forme de la courbe de croissance, la valeur moyenne de $v$ sur une période $\left(t_{1}, t_{2}\right)$ est estimée par : $v=\frac{\log \mathrm{X}_{2}-\log \mathrm{X}_{1}}{t_{2}-t_{1}}$; c'est cette estimation que nous adopterons ici. D'autre part, Hald (1960) considère que les taux spécifiques de croissance sont stochastiquement indépendants, ce qui pourra permettre dans un premier temps de traiter par les techniques de régression les modèles de la forme : $\frac{\mathrm{d}(\log \mathrm{X})}{\mathrm{d} t}=f(t)$.

b) Discrimination précoce des poules dw et des poules $\mathrm{Dw}$.

On a à discriminer deux populations $(d w, D w)$ à l'aide de 10 caractères ; ce problème a été traité par la méthode de la fonction discriminante linéaire de FISHER (I936); on peut se référer au travail de TOMASSONE (1963).

Chaque fonction calculée a été testée par un test de $\mathrm{F}$ :

où

$$
\begin{array}{r}
\mathrm{F}(p, \mathrm{~N}-p-\mathrm{I} ; \alpha)=\mathrm{D}^{2} \cdot \frac{n_{d w} \cdot n_{D w(\mathrm{~N}-p-1)}}{\mathrm{N} \cdot p \cdot(\mathrm{N}-2)} \\
\mathrm{N}=n_{d w}+n_{D w}=3^{2} \\
\mathrm{D}^{2}=\sum_{i=\mathrm{I}}^{p} b_{i} d_{i} \quad \text { avec } \quad d_{i}=\overline{\mathrm{X}}_{i(D w)}-\overline{\mathrm{X}}_{i(d w)}
\end{array}
$$

Le nombre de variable, $p$, peut varier de I à Io; l'efficacité de l'apport d'une nouvelle variable ou d'un groupe de variables se teste par un nouveau test de $F$; les variables considérées seront des poids corporels observés et/ou des vitesses spécifiques de croissance. Enfin, on donnera le taux de mauvaise classification pour la fonction discriminante retenue.

\section{II. - RÉSULTATS}

\section{A. - Description des deux populations}

(i) Poids corporel.

Le tableau I donne la moyenne, l'écart-type et le coefficient de variation du poids corporel hebdomadaire pour les groupes $\mathrm{C}_{1}, \mathrm{C}_{1}^{\prime}, \mathrm{C}_{2}, \mathrm{C}_{2}^{\prime}, \mathrm{C}_{3}, \mathrm{C}_{3}^{\prime}$.

La figure I indique 1'allure de la croissance pondérale moyenne des groupes $\mathrm{C}_{1}$ et $\mathrm{C}_{1}^{\prime}$. Les figures $2 a, 2 b, 2 c$ concrétisent 1'évolution de la variabilité du poids corporel selon l'âge. Le coefficient de variation du poids à l'éclosion des $d w$ est toujours inférieur à celui des $D w$ (environ $7 \mathrm{p}$. Ioo pour $d w$, Io p. Ioo pour $D w$ ) mais toujours plus élevé à une semaine (I I à I4 p. Ioo pour $d w, 7$ à Io p. roo pour $D w$ selon les groupes). Malgré la taille réduite des groupes, il convient de noter les points suivants :

- les coefficients de variation à deux semaines sont sensiblement égaux (Io à I2 p. roo);

- après 1'âge de deux semaines, le coefficient de variation du poids des poules $D w$ est plus élevé que celui des $d w$; on note en particulier une très sensible augmentation de ce coefficient à l'âge de 4 semaines dans le groupe $\mathrm{C}_{3}$;

- la variabilité minimum est observée à 4-5 semaines chez les $d w$ du groupe $\mathrm{C}_{3}^{\prime}$ (moins de $3 \mathrm{p}$. roo), en même temps que la variabilité maximum des $D w$ du groupe $\mathrm{C}_{3}$. Dans ces deux groupes, les variabilités sont sensiblement égales à. 9 semaines. 


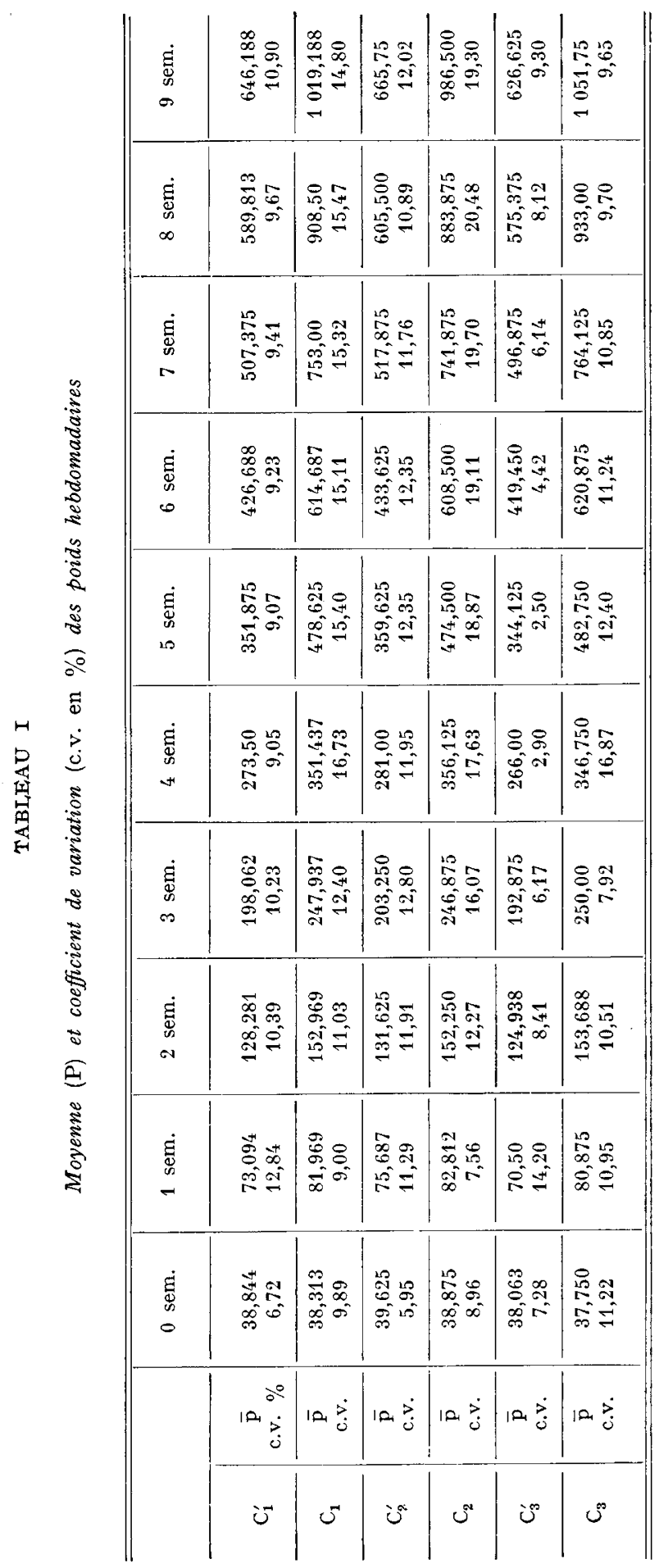




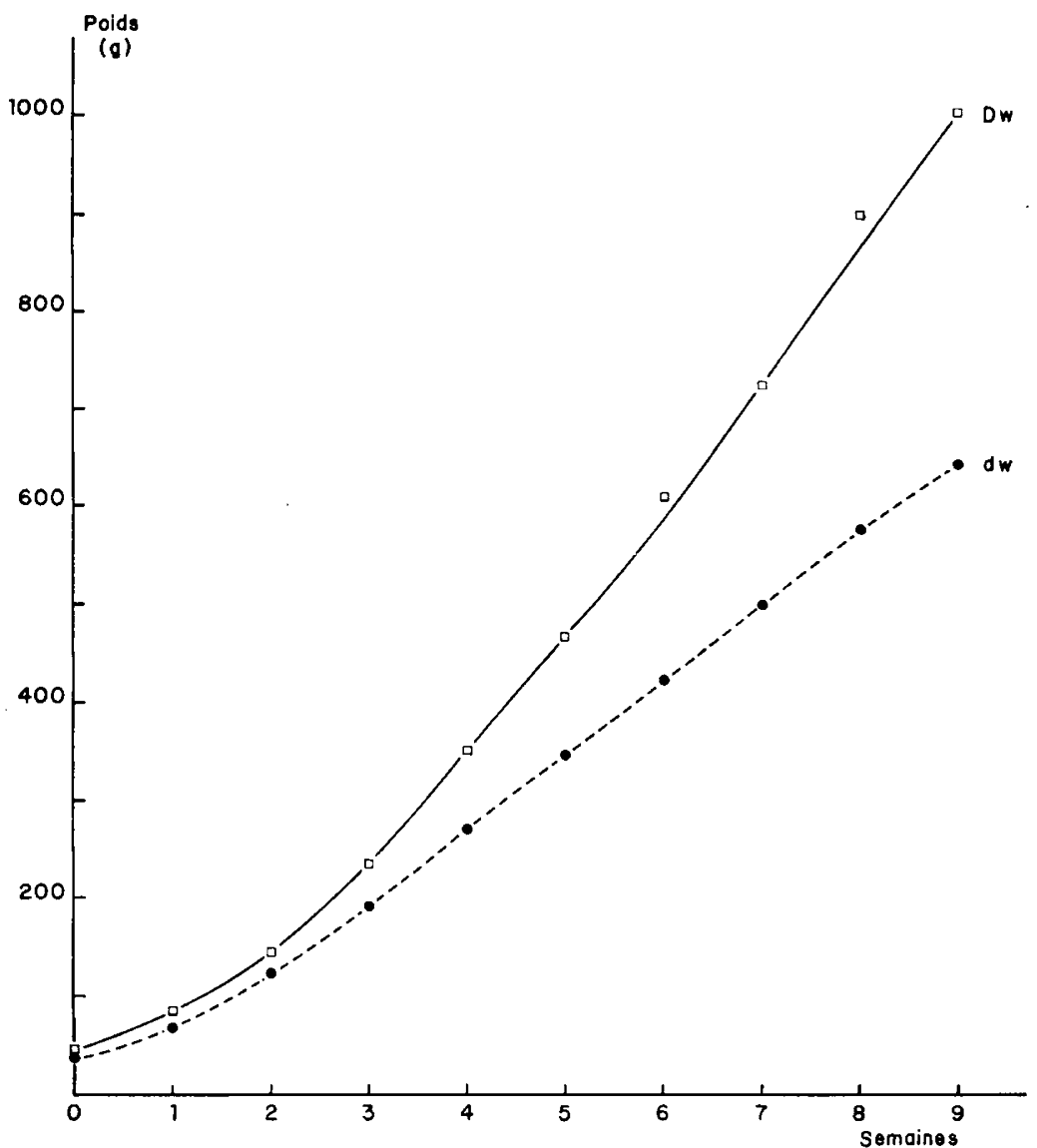

FIG. 1. - Courbe de croissance ponderale moyenne

\section{TABLEAU 2}

Coefficient de variation (en \%) calculé sur le logarithme du poids

\begin{tabular}{|c|c|c|c|c|c|c|c|c|c|c|}
\hline & 0 sem. & $1 \mathrm{sem}$ & 2 sem. & $3 \mathrm{sem}$ & 4 sem. & 5 sem. & $6 \mathrm{sem}$. & 7 sem. & 8 sem. & 9 sem. \\
\hline $\mathrm{C}_{1}^{\prime}$ & 1,83 & 3,28 & 2,21 & 2,01 & 1,61 & $1,5^{\prime}$ & 1,51 & 1,51 & 1,49 & 1,68 \\
\hline $\mathrm{C}_{1}$ & 2,71 & 2,08 & 2,21 & 2,25 & 3,01 & 2,61 & 2,45 & 2,41 & 2,38 & 2,27 \\
\hline $\mathrm{C}_{2}^{\prime}$ & 1,60 & 2,71 & 2,61 & 2,59 & 2,20 & 2,14 & 2,05 & 1,93 & 1,70 & 1,86 \\
\hline $\mathrm{C}_{2}$ & 2,41 & 1,69 & 2,45 & 2,94 & 3,04 & 3,18 & 3,08 & 3,06 & 3,08 & 2,91 \\
\hline $\mathrm{C}_{3}^{\prime}$ & 1,98 & 3,74 & 1,75 & 1,20 & 0,52 & 0,44 & 0,73 & 0,97 & 1,22 & 1,45 \\
\hline $\mathrm{C}_{3}$ & 3,08 . & 2,49 & 2,10 & 1,46 & 3,17 & 2,10 & 1,80 & 1,67 & 1,44 & 1,42 \\
\hline
\end{tabular}


(ii) Logarithme du poids corporel.

La figure 3 représente la courbe de croissance spécifique tracée d'après la moyenne des logarithmes des poids ; comme pour la courbe de croissance pondérale, les $D w$ sont toujours au-dessus des $d w$, mais l'utilisation du logarithme n'a eu aucun effet de linéarisation, ce qui indique un modèle sous-jacent de croissance assez complexe.

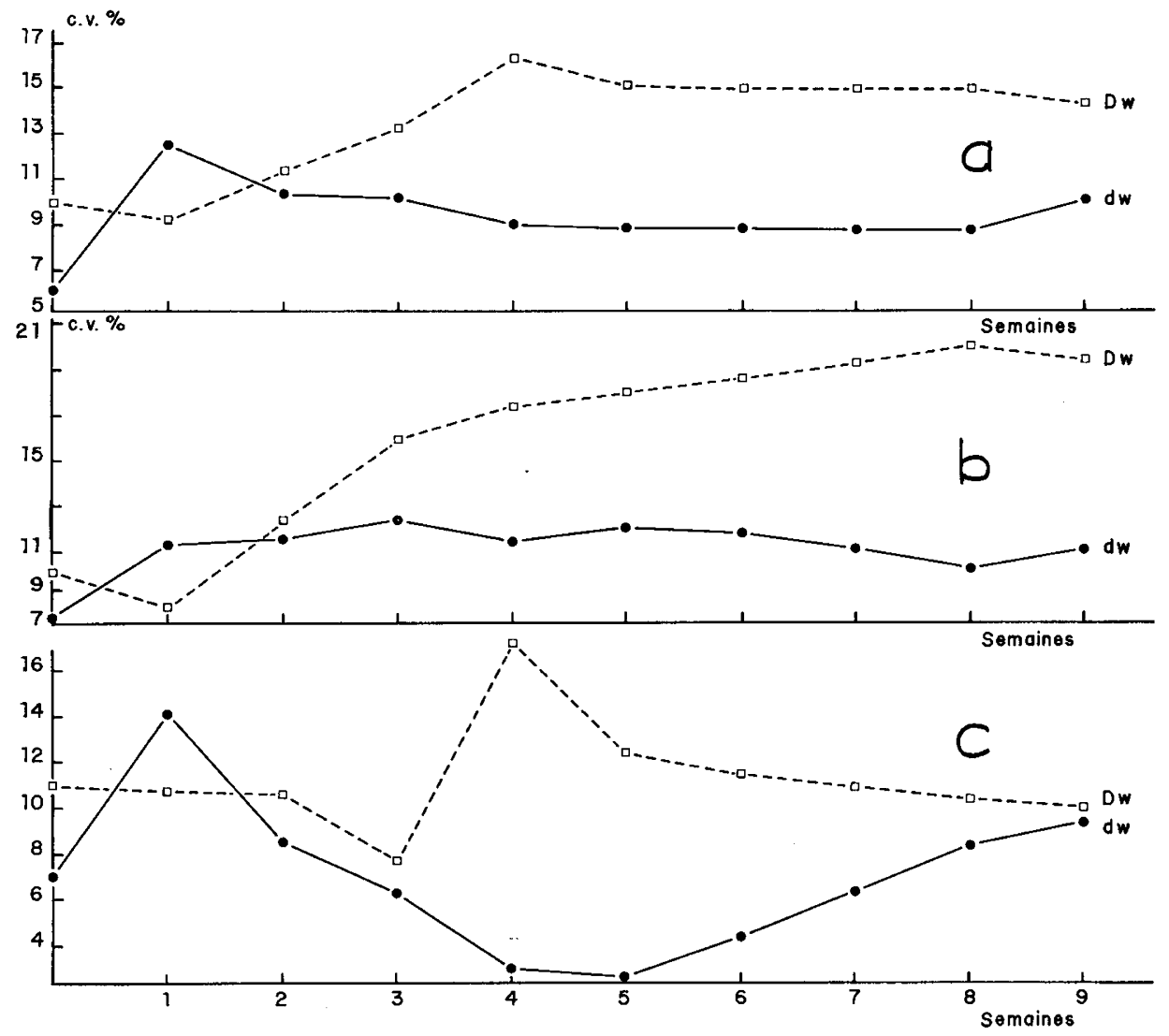

FIG. 2. - Variation du coefficient de variabilité $d u$ poids corporel (en p. Ioo) en fonction de l'âge en semaines

$$
\begin{aligned}
& a: \mathrm{C}_{1}, \mathrm{C}_{1}^{\prime} \\
& b: \mathrm{C}_{2}, \mathrm{C}_{2}^{\prime} \\
& c: \mathrm{C}_{3}, \mathrm{C}_{3}^{\prime}
\end{aligned}
$$

Le tableau 2 et la figure $4(a, b, c)$ indiquent l'évolution du coefficient de variation calculé sur le logarithme du poids ; ce coefficient est nettement réduit par l'utilisation de la transformation logarithmique, mais son allure de variation dans le temps est analogue.

(iii) Vitesse de croissance spécifique.

Le tableau 3 indique les valeurs de la vitesse de croissance spécifique hebdomadaire moyenne et son coefficient de variation. 
La figure 5 représente la décroissance de ce paramètre; on remarque que la vitesse spécifique de croissance des $D w$ est supérieure à celle des $d w$, particulièrement entre l'éclosion et la première semaine. Le coefficient de variation de ce paramètre est toujours élevé, particulièrement à 4-5 semaines pour les $D w$ et, jusqu'à l'âge de deux semaines, sa valeur est plus forte chez les $d w$.

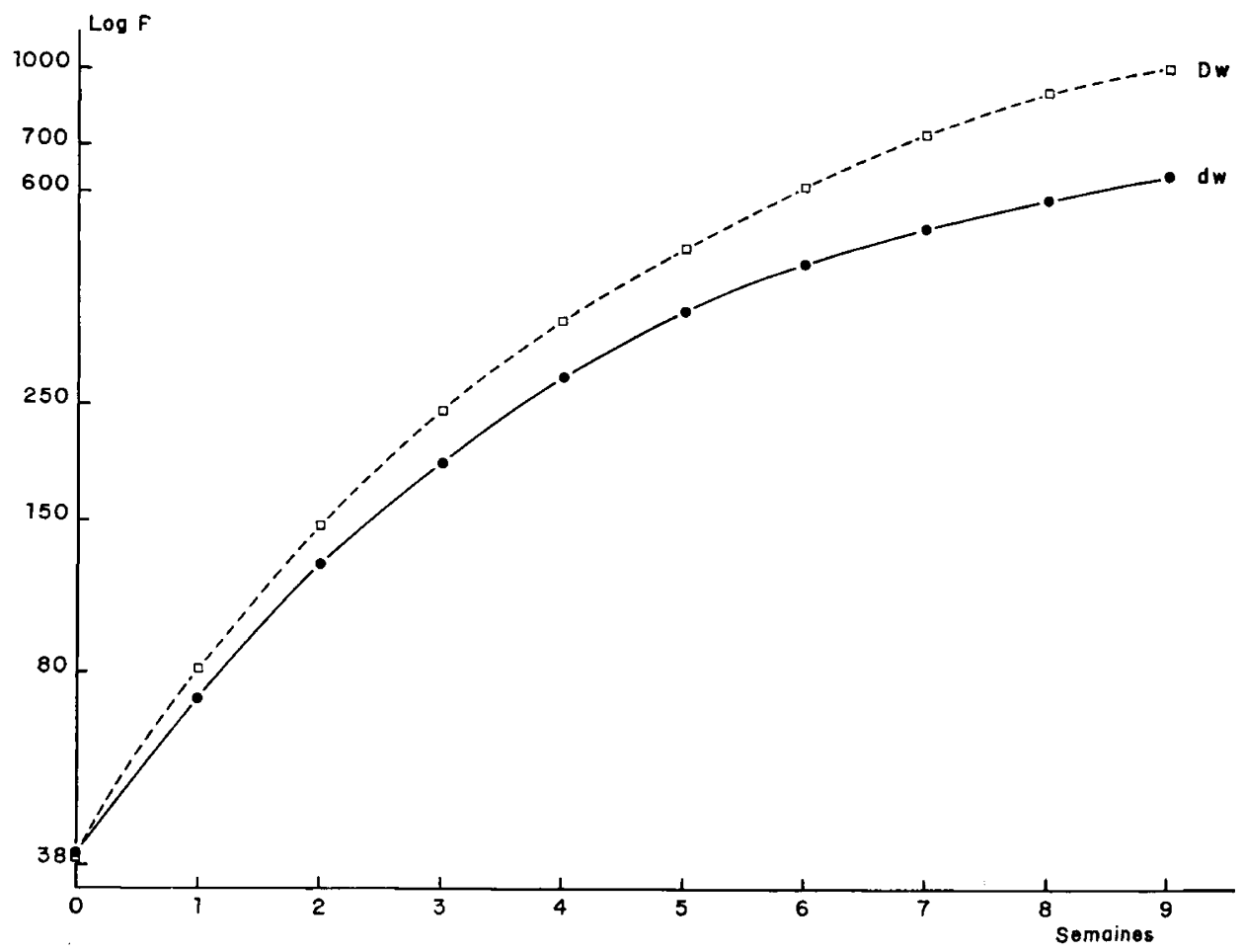

FIG. 3. - Courbe de croissance spécifique

(moyenne des logarithmes des poids par stade)

$$
\begin{array}{r}
D w \text { (groupe } \mathrm{C}_{1} \text { ) } \\
\text { d }
\end{array}
$$

On peut aussi calculer la vitesse de croissance spécifique moyenne entre l'éclosion et l'âge de deux jours :

$$
\begin{array}{lr}
C_{1}: & 0,029 \\
C_{1}^{\prime}: & 0,004 \\
C_{2}: & 0,046 \\
C_{2}^{\prime}: & 0,001 \\
C_{3}: & 0,011 \\
C_{3}^{\prime}: & 0,027
\end{array}
$$

(Les coefficients de variation sont compris entre 16 et $2 x$ p. roo)

On note une valeur de ce paramètre très faible pour les $d w$, avec, dans certains cas, une diminution du poids entre o et 2 jours ce qui se traduit par une vitesse spécifique négative. 


\begin{tabular}{|c|c|c|c|c|c|c|}
\hline 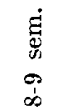 & 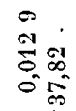 & $\begin{array}{l}10 \\
0 \\
0 \\
0 \\
0 \\
0\end{array}$ & 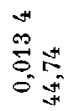 & 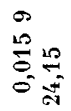 & 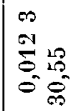 & 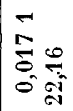 \\
\hline 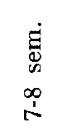 & $\begin{array}{l}10 \\
\overline{1}=0 \\
0 \\
0 \\
0.00\end{array}$ & $\begin{array}{l}\infty \\
0 \\
\stackrel{1}{0} \\
\stackrel{0}{0} \\
0\end{array}$ & $\begin{array}{l}10 \\
\text { ov } \\
\text { ov } \\
0.5 \\
0\end{array}$ & $\begin{array}{l}\infty \\
\stackrel{+}{*} \infty \\
0 \\
0\end{array}$ & 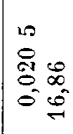 & 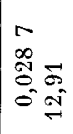 \\
\hline 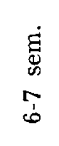 & 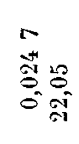 & $\begin{array}{l}\infty \\
\infty \\
\infty \\
0 \\
0 \\
0 \\
0 \\
0\end{array}$ & 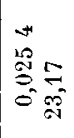 & $\begin{array}{l}1 \\
\infty \\
\infty \\
0 \\
0 \\
0 \\
0\end{array}$ & 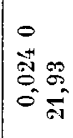 & 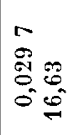 \\
\hline 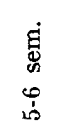 & $\begin{array}{l}\text { so } \\
\text { Tิ } \\
0 \\
0 \\
0\end{array}$ & $\begin{array}{l}\infty \\
20 \\
00 \\
0 \\
0 \\
0\end{array}$ & 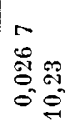 & $\begin{array}{l}10 \\
10 \\
00 \\
0 \\
0 \\
0 \\
0 \\
0\end{array}$ & $\begin{array}{l}\infty \\
\infty \\
\infty \\
0 \\
0 \\
0 \\
0 \\
0\end{array}$ & 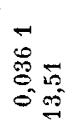 \\
\hline 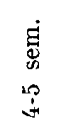 & 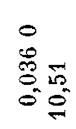 & $\underbrace{+\infty}_{0} \underset{0}{=}$ & $\begin{array}{l}0 \\
10 \\
00 \\
0 \\
0 \\
0 \\
0\end{array}$ & $\begin{array}{l}0 \\
0 \\
0 \\
0 \\
0 \\
0\end{array}$ & 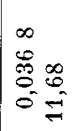 & 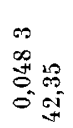 \\
\hline 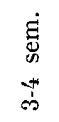 & $\begin{array}{l}\infty \\
00 \\
0 \\
0 \\
0 \\
0 \\
0\end{array}$ & $\begin{array}{l}0 \\
\infty \\
\infty \\
0 \\
0 \\
0 \\
0 \\
0\end{array}$ & $\begin{array}{l}10 \\
0 \\
0 \\
0 \\
0 \\
0\end{array}$ & $\begin{array}{l}0 \\
\overrightarrow{20} \\
0 \\
0 \\
0\end{array}$ & 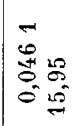 & 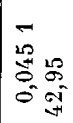 \\
\hline 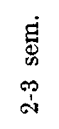 & $\begin{array}{l}\vec{\gamma} \\
\hat{0} \\
0 \\
0 \\
0\end{array}$ & $\begin{array}{l}\overrightarrow{8} 0 \\
80 \\
0 \\
0\end{array}$ & $\begin{array}{l}0 \\
\overrightarrow{8} \\
\dot{E} \\
0 \\
0\end{array}$ & $\begin{array}{l}\overrightarrow{1} \\
\infty \\
\stackrel{0}{0} \\
0 \\
0\end{array}$ & 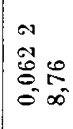 & $\begin{array}{l}\infty \\
0 \\
8 \\
0 \\
0 \\
0\end{array}$ \\
\hline 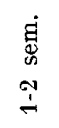 & $\begin{array}{l}\infty \\
0 \\
0 \\
0 \\
0 \\
0\end{array}$ & $\begin{array}{ll}\bar{D} & \\
\mathscr{D} & 0 \\
0 & 0 \\
0 & 0\end{array}$ & $\begin{array}{l}\infty \\
\infty \\
0=0 \\
0=0 \\
0=\infty\end{array}$ & $\begin{array}{l}+1 \\
0 \\
5 \\
0 \\
0 \\
0 \\
0\end{array}$ & $\begin{array}{l}\infty \\
\infty \\
01 \\
0 \\
0 \\
0 \\
0 \\
0 \\
0\end{array}$ & $\begin{array}{l}\infty \\
\overline{8} \\
0.0 \\
0.00\end{array}$ \\
\hline $\begin{array}{l}\text { घ્ } \\
\text { w } \\
\vec{b}\end{array}$ & $\begin{array}{l}+ \\
\infty \\
0 \\
0 \\
0 \\
0 \\
0\end{array}$ & $\left\{\begin{array}{l}n \\
\infty \\
0 \\
0 \\
0 \\
0 \\
0\end{array}\right.$ & $\begin{array}{l}\infty \\
\vec{\delta} \\
0 \\
0\end{array}$ & $\begin{array}{l}\infty \\
\infty \\
0 \\
0 \\
0 \\
0 \\
0 \\
0\end{array}$ & $\begin{array}{l}\infty \\
0 \\
\infty \\
0 \\
0 \\
0 \\
0\end{array}$ & $\begin{array}{l}0 \\
8 \\
\stackrel{0}{0} \\
0 \\
0 \\
0\end{array}$ \\
\hline & $\stackrel{\overrightarrow{0}}{\vec{c}}$ & $\stackrel{\overrightarrow{0}}{\dot{0}} \dot{0}$ & 完 & $\stackrel{\overrightarrow{0}}{\vec{z}} \dot{\vec{c}}$ & $\stackrel{\overrightarrow{0}}{\sum^{2}}$ & 客完 \\
\hline & v & 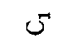 & ن̃ & w & $u^{\infty}$ & $\mho^{\infty}$ \\
\hline
\end{tabular}

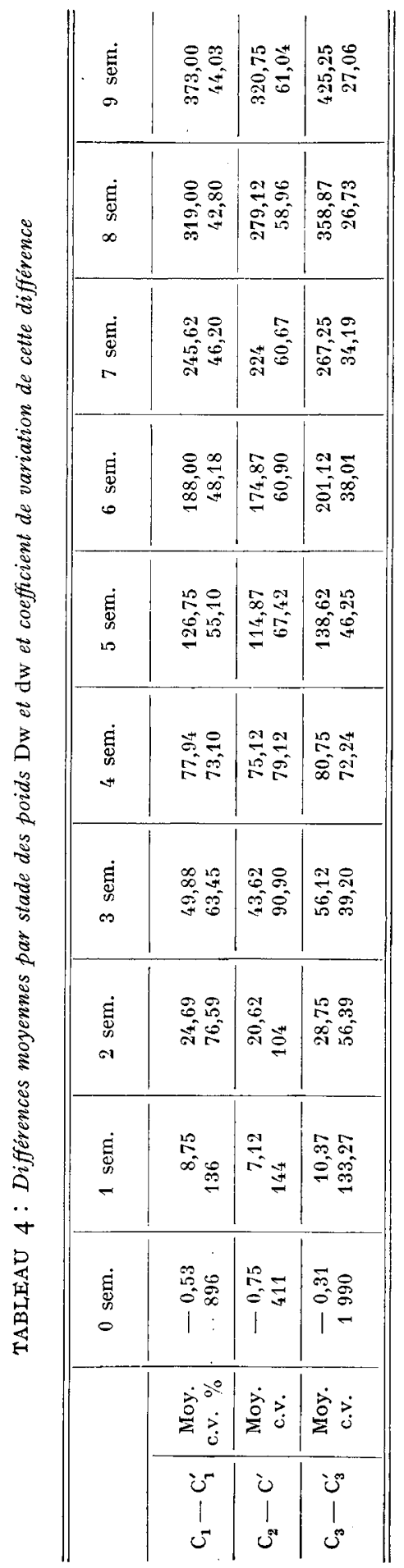




\section{B. - Discrimination des Dw et des dw}

\section{(i) Études préliminaires.}

On a d'abord étudié la forme des distributions des poids à chaque stade et leur recouvrement ; les distributions des poids des $d w$ sont moins asymétriques que celles des $D w$, en particulier à 4 semaines (coefficient $\beta_{1}=5 \cdot \mathrm{IO}^{-4}$ ). L'utilisation du test du $\chi^{2}$ montre un recouvrement non significatif à $I$ p. Ioo à partir de 5 semaines.

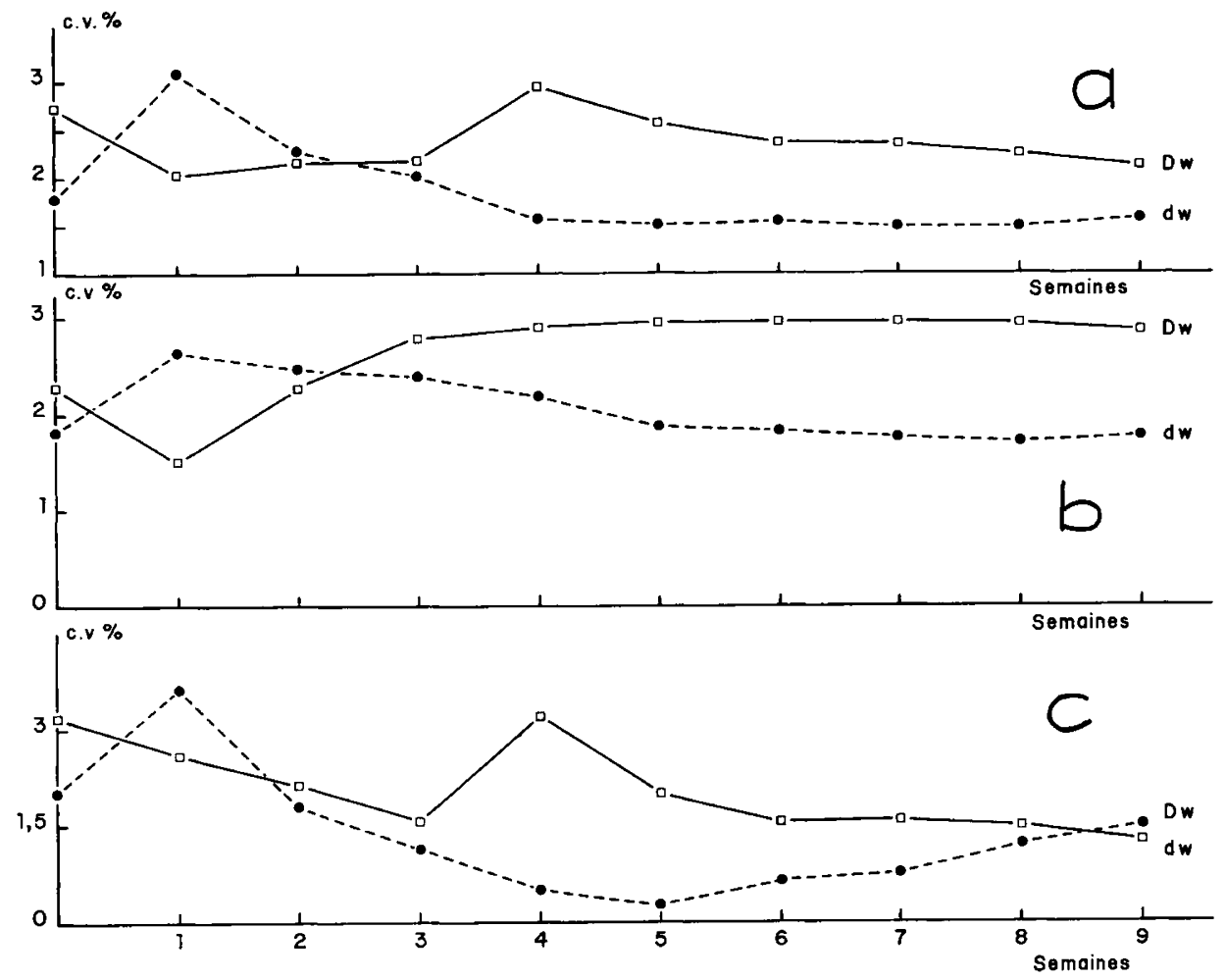

FIG. 4. - Variation du coefficient de variabilité (en p. Ioo) du logarithme du poids corporel en fonction de l'âge en semaines

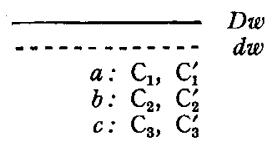

On a ensuite comparé les poids corporels hebdomadaires entre groupes par analyse de variance à un facteur contrôlé (l'âge) ; les différences entre $d w$ et $D w$ sont significatives à I p. I 000 dès l'âge de 4 semaines. Le tableau 4 indique, pour chaque stade, la moyenne et la variabilité des différences du type $\overline{\mathrm{P}_{\mathrm{C}_{\mathfrak{i}}}-\mathrm{P}_{\mathrm{C}_{\mathrm{i}}^{\prime}}}(i=\mathrm{I}, 2,3)$; la figure 6 montre la variation de ces différences au cours de la croissance; on

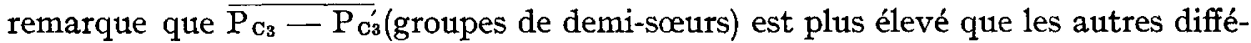
rences, ce qui est confirmé par l'analyse de variance. 


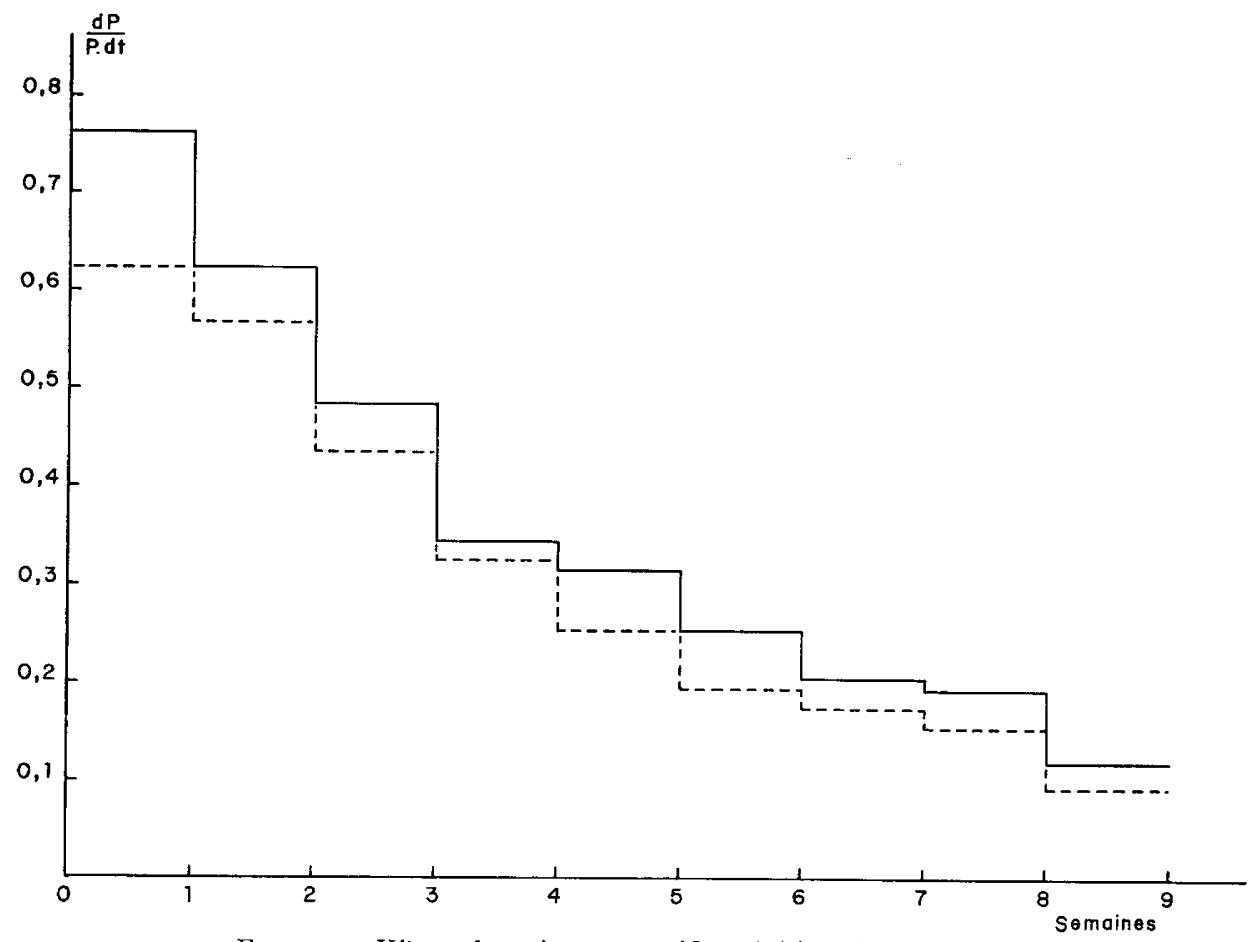

FIG. 5. - Vitesse de croissance spécifique hebdomadaire moyenne

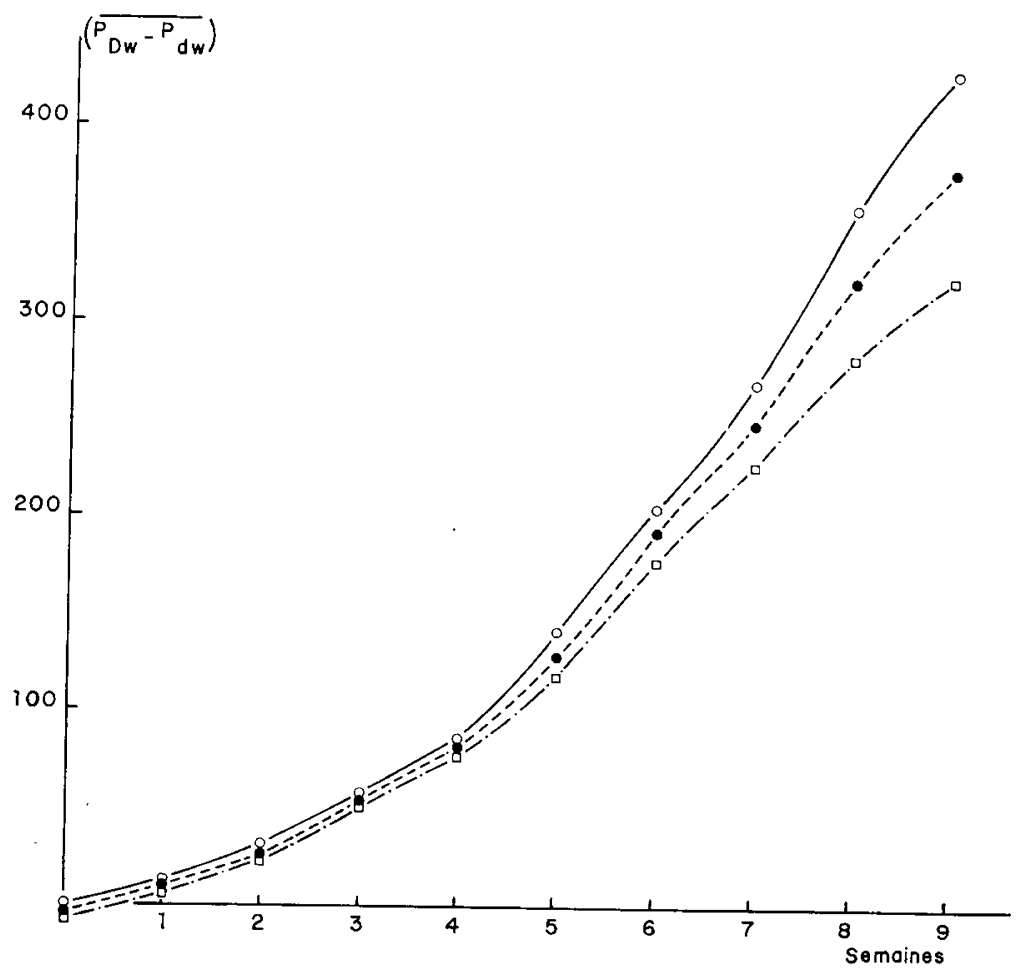

FIG. 6. - Moyenne des différences entre Dw et $\mathrm{dw}$ des poids par stade selon les groupes $C_{1}, C_{2}, C_{3}$

$\mathrm{P}_{\mathrm{C}_{1}}-\mathrm{P}_{\mathrm{C}_{1}^{\prime}} \quad \cdots \ldots . . . . .-\mathrm{P}_{\mathrm{C}_{2}}-\mathrm{P}_{\mathrm{C}_{2}^{\prime}}$ 
(ii) Fonction discriminante linéaire (discrimination entre $C_{\mathbf{1}}$ et $C_{\mathbf{1}}^{\prime}$ ).

La fonction suivante a été retenue.

$$
\mathrm{Y}=\text { I0,9I } \mathrm{X}_{1}+0,056 \mathrm{X}_{2}
$$

où $\mathrm{X}_{1}$ est la vitesse spécifique de croissance hebdomadaire moyenne entre l'éclosion et une semaine, c'est-à-dire :

$$
\mathrm{X}_{1}=\log \left(\frac{\text { Poids corporel à une semaine }}{\text { Poids corporel à l'éclosion }}\right)
$$

$\mathrm{X}_{2}$ est le poids à 2 semaines.

Cette fonction est significative au seuil I p. I 000 :

$$
\mathrm{F}(2,29)=\mathrm{I} 76,80 \quad \mathrm{D}^{2}=45,72
$$

Le taux de mauvaise classification est de 2 p. Ioo ; c'est-à-dire que 2 p. Ioo des individus discriminés à l'aide de cette fonction seront assignés au " mauvais " groupe. Les $d w$ seront discriminés des $D w$ d'après les critères suivants :

- si, pour une poule on calcule $\mathrm{Y}>\mathrm{I} 5,36$, elle sera classée en $D w$

- si, on calcule $\mathrm{Y}<\mathrm{I} 4,38$, on la classera en $d w$

- aucune classification ne sera donnée pour $14,38 \leqslant Y \leqslant 15,36$.

Parmi toutes les fonctions calculées en utilisant les poids de l'éclosion à 4 semaines et les vitesses spécifiques dans cette période, la fonction proposée ici est optimale vis-à-vis de la précocité de la discrimination et vis-à-vis de sa signification statistique. Ceci peut s'expliquer en partie si on se souvient que la différence entre les vitesses spécifiques de croissance des $D w$ et $d w$ est maximum entre o et une semaine et que les poids corporels ont à deux semaines une variabilité très comparable.

\section{III. - DISCUSSION}

Cette étude est très incomplète et n'a qu'un but descriptif ; son objectif principal (calcul d'une fonction discriminante) semble avoir été atteint, puisque nous avons pu la contrôler sur des données fournies par RICARD. C'est d'ailleurs sur cet échantillon comprenant plus de 200 animaux que nous compléterons l'étude comparative de la croissance des $d w$ et $D w$ dans deux directions :

- par analyse multivariate (analyse factorielle et analyse des correspondances), Milizier essaiera de définir les phases de la croissance des poules naines et normales ;

- puis recherche d'un modèle susceptible de rendre compte de la succession de ces cycles et de leur importance respective chez les $D w$ et $d w$.

En effet, d'après les données présentes, les poules naines sont caractérisées par une vitesse spécifique de croissance sensiblement plus faible que celle des poules normales, en particulier lors des premières semaines de vie postnatale; la régression du poids observée entre l'éclosion et deux jours (perte du sac vitellin) est très marquée chez les $d w$. Tout se passe comme si les poules naines ne pouvaient compenser le retard en vitesse qui leur est infligé dès l'éclosion; comme on ne dispose pas de données concernant les embryons, il est important de vérifier si les courbes de croissance postnatale pondérale des $d w$ et $D w$ sont : 
- soit simplement caractérisées par une différence croissante dans le temps;

- soit caractérisées par des phases différentes, c'est-à-dire décalées dans le temps et sur les poids.

Le calcul des régressions polynomiales individuelles par les polygones orthogonaux ne permet de tirer aucune conclusion : tous les individus ont une croissance assez bien décrite par des polynomes de la forme : $\mathrm{P}_{i}=a_{i}+b_{i} t+\mathrm{C}_{i} t^{2}$, les coefficients étant significativement plus élevés pour les $D w$.

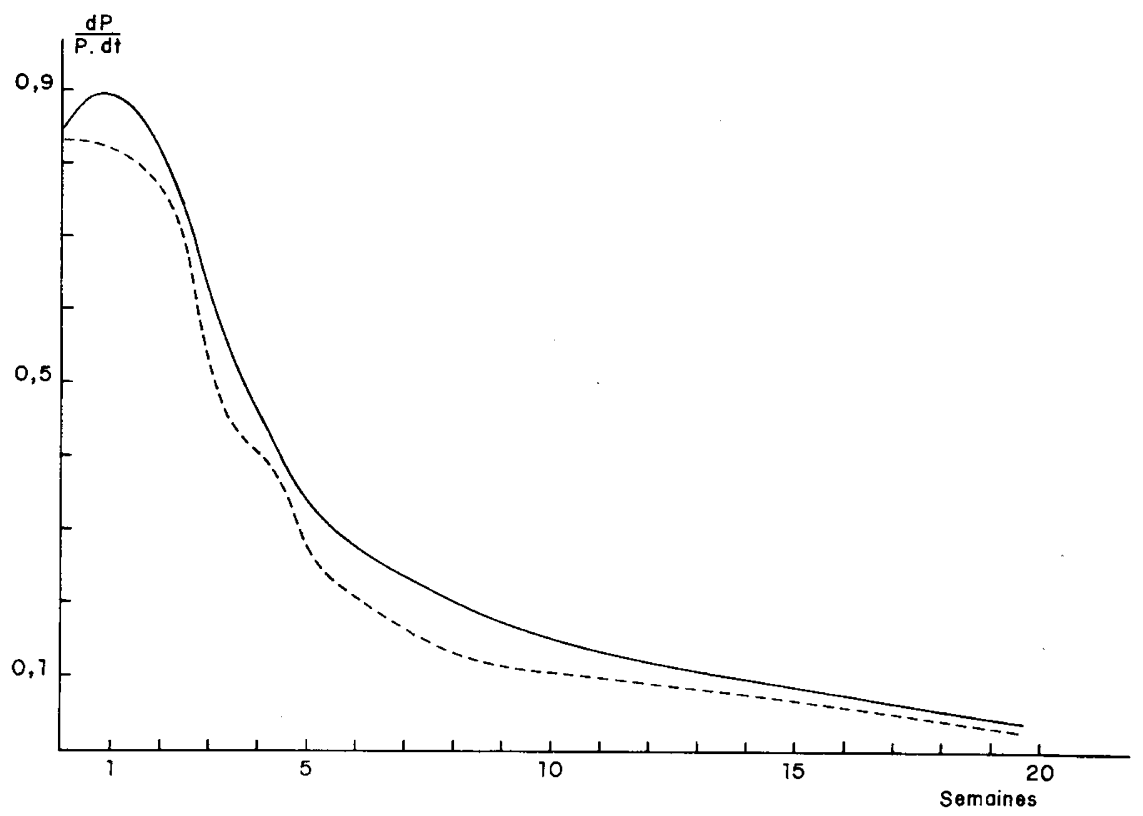

FIG. 7. - Courbes de vitesse de croissance spécifique

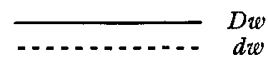

Cependant, une étude analytique en cours sur un échantillon de taille plus importante fourni par RICARD et concernant des pesées hebdomadaires effectuées jusqu'à 20 semaines semble confirmer une différence notable entre $D w$ et $d w$ pour la vitesse spécifique de croissance; les allures générales approximatives sont données à la figure $7:$ les formes des courbes ne sont pas simples, et c'est leur analyse comparative que nous devrons aborder.

Reçu pour publication en décembre 1971.

\section{SUMMARY}

BIOMETRIC DESCRIPTION OF THE WEIGHT GROWTH OF DWARF AND NORMAL, HENS : EARLY DISCRIMINATION ON BODY WEIGHT

A sample of 32 hens ( $16 d w$ and I $6 D w$ ) was weighed weekly from hatching to 9 weeks. Variability of body weight was first studied. It was always higher in the $D w$ hens after 2 weeks. Specific growth rate of $D w$ hens was higher than that of $d w$ hens, particularly during the first 
postnatal week. A linear discriminant function was computed, thus permitting the $d w$ hens to be discriminated from the $D w$ hens at the second week according to specific average growth rate in the first week $\left(\mathrm{X}_{1}\right)$ and the body weight at 2 weeks $\left(\mathrm{X}_{2}\right)$, with a bad classification rate of 2 p. Ioo.

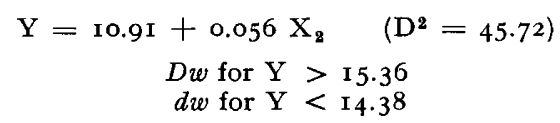

\section{RÉFÉRENCES BIBLIOGRAPHIQUES}

Fisher R. A., 192r. Some remarks on the methods formulated in a recent article on the " quantitative analysis of plant growth ". Ann. appl. Biol., 7, 367-372.

Fisher R. A., 1936. The use of multiple measurements in taxonomic problems. Ann. Eugen., 7, I79-I88.

HALD A., 1960. Statistical theory with engineering applications. Wiley, New York.

Tomassone R., 1963. Application des fonctions discriminantes à des problemes biométriques. Ann. Éc. nat. Eaux, Forêts, Nancy, 20, 585-6I7. 